\title{
An Experimental Study of Mixed-Path Groundwave Propagation ${ }^{1}$
}

\author{
S. W. Maley and H. Ottesen \\ Contribution From Electrical Engineering Department, University of Colorado, Boulder, Colo.
}

(Received April 6, 1964)

\begin{abstract}
A carefully controlled laboratory model study was made of electromagnetic propagation between two antennas on the surface of an imperfectly conducting ground plane over a path on which the parameters of the ground abruptly change at some point along the path. The experiment was done at X-band frequencies $(10 \mathrm{Gc} / \mathrm{s})$; the mixed path investigated was partly over water and partly over a metallic conductor. The experimental results were compared with theoretical work by J. R. Wait; the comparison indicates that Wait's theory agrees well with the experimental data.
\end{abstract}

\section{Introduction}

Groundwave propagation over a path along which the ground parameters abruptly change from one set of values to another has been studied theoretically by many persons [Suda, 1954; Kirke, 1949; Millington, 1949; Clemmow, 1953; Bremmer, 1954; Wait, 1956]. J. R. Wait's [1956] paper surveys the previous literature on the subject and gives a formulation of the problem in terms of an integral equation. A zero order solution was given for this equation. The solution was compared with earlier theoretical work and in particular with the semiempirical formula of Millington [1949], the comparison showed good agreement with Millington. The solution was also compared with some experimental data due to P. A. Field, this comparison also showed good agreement; but the experimental data available were restricted to a narrow range of distances. The experimental work reported in this paper was conducted at microwave frequencies $(10 \mathrm{Gc} / \mathrm{s})$, in the laboratory, and under carefully controlled conditions. The mixed path was partly over water and partly over a metallic conductor. The experimental results are compared with calculations from Wait's theoretical solution. This experimental investigation is not an exact scale model of a typical mixed path over the earth at lower frequencies; however, if Wait's solution were successful at predicting the propagation for this case, it could be applied with somewhat greater confidence to cases with other frequencies and ground parameters.

1 This work was supported in part by the University of Colorado, Council on Research and in part by the Air Force Cambridge Research Laboratories Contracts AFCRL-62-745, and AFCRL-63-583.

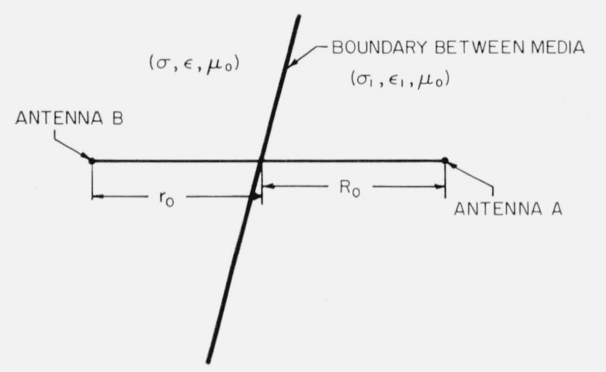

FiguRE 1. Top view of mixed propagation path.

\section{Theory}

The problem considered by Wait is shown in figure 1. A cylindrical coordinate system is used. The $z=0$ plane coincides with the surface of the imperfectly conducting ground, and the origin is located at antenna B. The ground parameters to the left of the boundary are $\sigma, \epsilon, \mu_{0}$, where $\mu_{0}$ is the permeability of free space, $\epsilon$ is permittivity and $\sigma$ is conductivity; and to the right of the boundary they are $\sigma_{1}, \epsilon_{1}, \mu_{0}$.

If the ground were homogeneous and with parameters $\sigma, \epsilon, \mu_{0}$, then the tangential magnetic field, $H_{\iota}$, at $A$ due to a current of $I_{b}$ in antenna $\mathrm{B}$ would be given by [Wait, 1956]. ${ }^{2}$

${ }^{2}$ MKS units will be used throughout this paper, and the time dependence will be assumed as_e $e^{i \omega t}$. 
$H_{t}=\frac{i \beta I_{b} h_{b}}{2 \pi\left(r_{0}+R_{0}\right)} e^{-i \beta\left(r_{0}+R_{0}\right)}\left[1+\frac{1}{i \beta\left(r_{0}+R_{0}\right)}\right] F\left(r_{0}+R_{0}, \eta\right)$

where $i=\sqrt{-1}, \beta=2 \pi / \lambda, \lambda$ is free space wavelength, $h_{b}$ is the effective height of antenna $\mathrm{B}$, and $\eta$ is the surface impedance, for a plane wave at grazing incidence, of the ground. $\eta$ is given by

$$
\eta=120 \pi \frac{i \beta}{\alpha}\left[1+\frac{\beta^{2}}{\alpha^{2}}\right]^{1 / 2} ;
$$

in this relation, $\alpha$ is defined by

$$
\alpha=\left(i \sigma \mu_{0} \omega-\epsilon \mu_{0} \omega^{2}\right)^{1 / 2},
$$

where $\omega$ is the angular frequency. The factor, $F\left(r_{0}+R_{0}, \eta\right)$, in (1) is the groundwave attenuation function as defined by Norton [1936], it is given by

where

$$
F(r, \eta)=1-i 2 p^{1 / 2} e^{-p} \int_{q=i p^{1 / 2}}^{\infty} e^{-q^{2}} d q,
$$

$$
p=-\frac{i \beta r}{2}\left(\frac{\eta}{120 \pi}\right)^{2} \text {. }
$$

For the case in which the ground is inhomogeneous, as shown in figure 1, (1) may be replaced by the relation

$$
\begin{aligned}
H_{t}=\frac{i \beta I_{b} h_{b}}{2 \pi\left(r_{0}+R_{0}\right)} e^{-i \beta\left(r_{0}+R_{0}\right)} & \\
& {\left[1+\frac{1}{\beta\left(r_{0}+R_{0}\right)}\right] F^{\prime}\left(r_{0}+R_{0}, \eta, \eta_{1}\right) . }
\end{aligned}
$$

$F^{\prime}\left(r_{0}+R_{0}, \eta, \eta_{1}\right)$ is an unknown groundwave attenuation factor for the mixed path for which part of the path, $r_{0}$, is over a surface for which $\eta$ is given by (2), and the other part of the path, $R_{0}$, is over a surface for which the surface impedance for a plane wave at grazing incidence is given by (2) and (3) with $\sigma$ and $\epsilon$ replaced by $\sigma_{1}$ and $\epsilon_{1}$. Wait formulates an integral equation for $F^{\prime}\left(r_{0}+R_{0}, \eta, \eta_{1}\right)$ and gives as a zero order solution,

$$
\begin{aligned}
F^{\prime}\left(r_{0}+R_{0}, \eta, \eta_{1}\right)= & F\left(r_{0}+R_{0}, \eta\right)+i\left(\frac{p_{0}}{\pi}\right)^{1 / 2}\left(1-\frac{1}{\sqrt{K}}\right) \\
& \int_{0}^{R_{0}} \frac{F\left(r_{0}+r, \eta\right) F\left(R_{0}-r, \eta_{1}\right)}{\sqrt{\left(R_{0}+r\right)\left(r_{0}-r\right)}} d r,
\end{aligned}
$$

where

$$
\begin{gathered}
p_{0}=-\frac{i \beta\left(r_{0}+R_{0}\right)}{2}\left(\frac{\eta}{120 \pi}\right)^{2}, \\
K=\frac{\sigma_{1}+i \omega \epsilon_{1}}{\sigma+i \omega \epsilon}
\end{gathered}
$$

and

$$
V=\frac{R_{0}}{r_{0}+R_{0}}
$$

This experimental investigation is concerned with a path for which $\epsilon / \epsilon_{0}=49, \sigma / \omega \epsilon_{0}=34$ and $\sigma_{1}=\infty$, where $\epsilon_{0}$ is the permittivity of free space $\left(\epsilon_{0}=10^{-9} / 36 \pi\right)$. For these values, (7) can be expressed in the form

$$
\begin{aligned}
& F^{\prime}\left(r_{0}+R_{0}, \eta, \eta_{1}\right)=F\left(r_{0}+R_{0}, \eta\right) \\
&+i\left(\frac{p_{0}}{\pi}\right)^{1 / 2} \int_{0}^{R_{0}} \frac{F\left(r_{0}+r, \eta\right)}{\sqrt{\left(r_{0}+r\right)\left(R_{0}-r\right)}} d r .
\end{aligned}
$$

Using (4), (5), and (11), the quantity $\left|F^{\prime}\left(r_{0}+R_{0}, \eta, \eta_{1}\right)\right|$ was calculated by a numerical integration procedure for values of $r_{0}+R_{0}$ ranging from 8.3 to 66.7 wavelengths. The calculated values are plotted as solid lines in figures 2 to 6 .

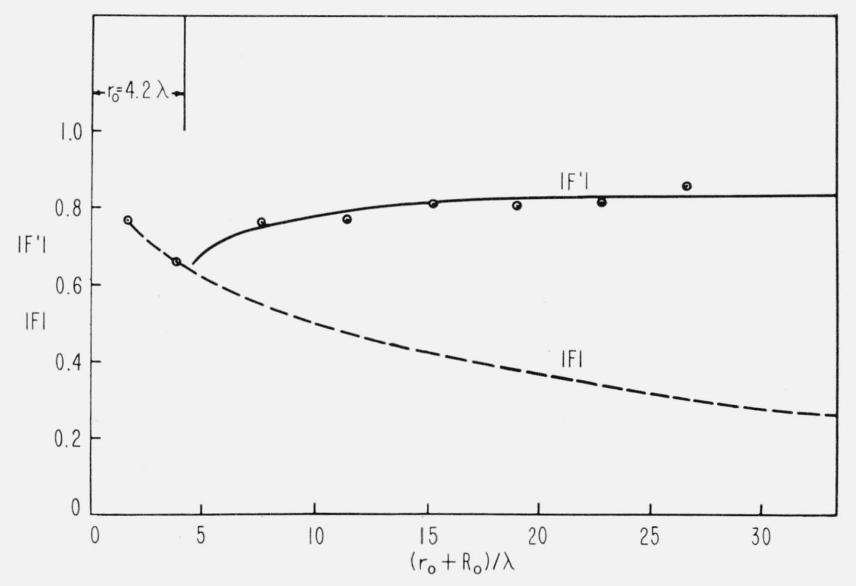

Figure 2. Mixed-path groundwave attenuation function, $\left|\mathrm{F}^{\prime}\right|$, versus $\left(\mathrm{r}_{0}+\mathrm{R}_{0}\right) / \lambda$ for $\mathrm{r}_{0}=4.2 \lambda$.

A value of $\left(r_{0}+R_{0}\right) / \lambda$ less than $r_{0} \lambda$ implies that $R_{0} / \lambda$ is negative or that antenna $\mathrm{A}$ is to the left of the boundary (ref. fig. 1).

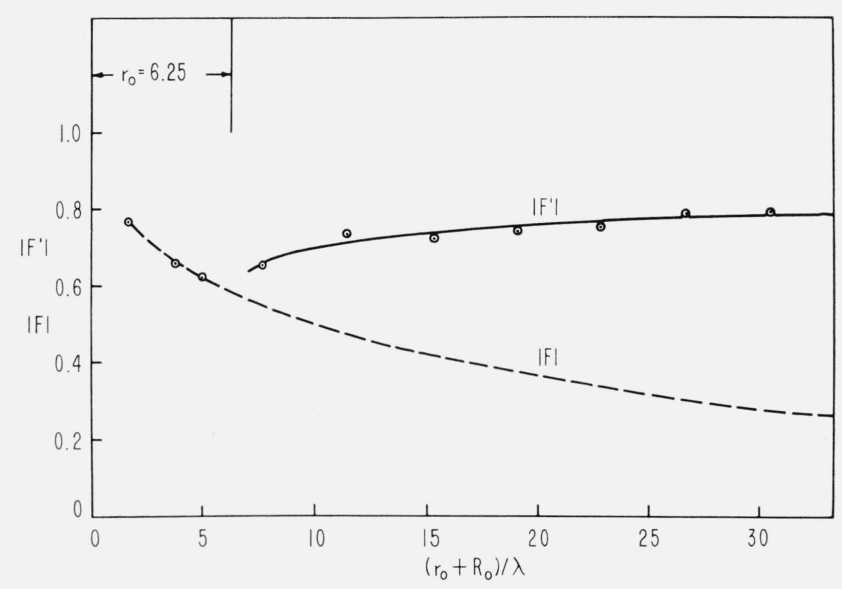

Figure 3. Mixed-path groundwave attenuation function, $\left|\mathrm{F}^{\prime}\right|$, versus $\left(\mathrm{r}_{0}+\mathrm{R}_{0}\right) / \lambda$ for $\mathrm{r}_{0}=6.25 \lambda$.

A value of $\left(r_{0}+R_{0}\right) / \lambda$ less than $r_{0} / \lambda$ implies that $R_{0} / \lambda$ is negative or that antenna $\mathrm{A}$ is to the left of the boundary (ref. fig. 1). 


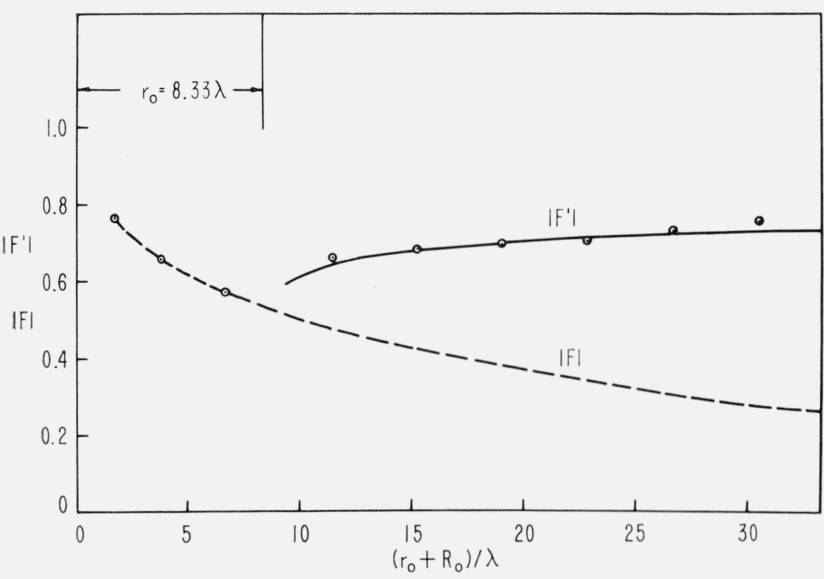

Figure 4. Mixed-path groundwave attenuation function, $\left|\mathrm{F}^{\prime}\right|$, versus $\left(\mathrm{r}_{0}+\mathrm{R}_{0}\right) / \lambda$ for $\mathrm{r}_{0}=8.33 \lambda$.

A value of $\left(r_{0}+R_{0}\right) / \lambda$ less than $r_{0} / \lambda$ implies that $R_{0} / \lambda$ is negative or that'antenna $\mathrm{A}$ is to the left. of the boundary (ref. fig. 1).

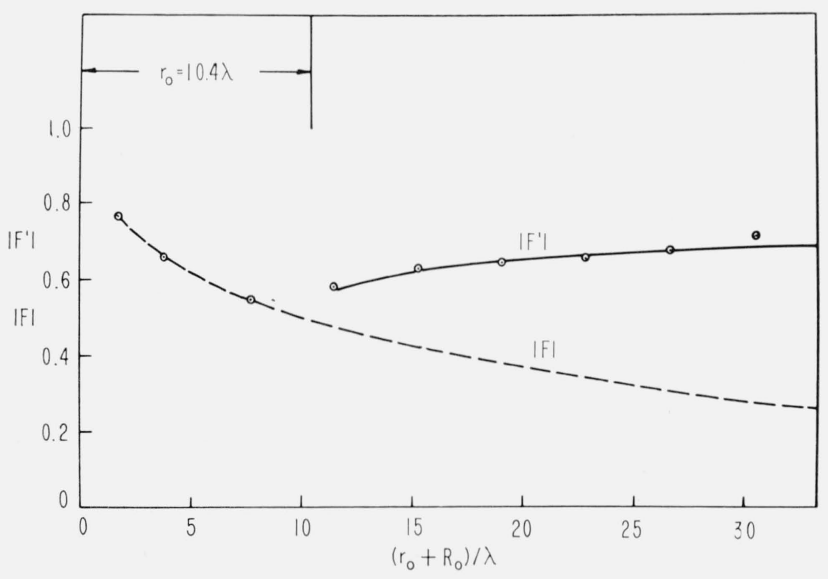

Figure 5. Mixed-path groundwave attenuation function, $\left|\mathrm{F}^{\prime}\right|$, versus $\left(\mathrm{r}_{0}+\mathrm{R}_{0}\right) / \lambda$ for $\mathrm{r}_{0}=10.4 \lambda$.

A value of $\left(r_{0}+R_{0}\right) / \lambda$ less than $r_{0} / \lambda$ implies that $R_{0} / \lambda$ is negative or that antenna $\mathrm{A}$ is to the left of the boundary (ref. fig. 1).

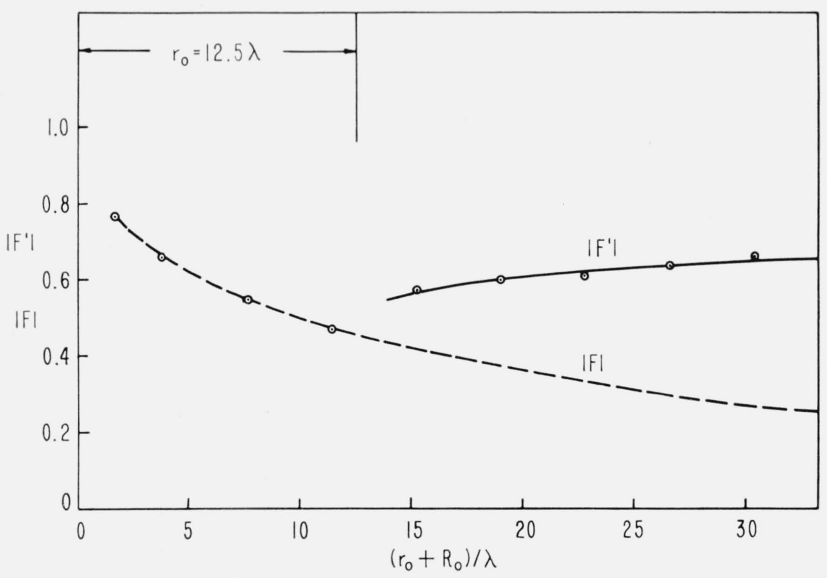

Figure 6. Mixed-path groundwave attenuation function, $\left|\mathrm{F}^{\prime}\right|$, versus $\left(\mathrm{r}_{0}+\mathrm{R}_{0}\right) / \lambda$ for $\mathrm{r}_{0}=12.5 \lambda$.

A value of $\left(r_{0}+R_{0}\right) / \lambda$ less than $r_{0} / \lambda$ implies that $R_{0} / \lambda$ is negative or that antenna $\mathrm{A}$ is to the left of the boundary (ref. fig. 1).

\section{Experimental Investigations}

The experimental measurements were made at $\mathrm{X}$-band frequencies $(10 \mathrm{Gc} / \mathrm{s})$ in the laboratory. The transmitting antenna was a short base fed vertical monopole located approximately at the center of a wooden water tank and was positioned just above the water surface. The water tank was 120 wavelengths square and was surrounded by absorbing material to prevent reflections. An aluminum sheet was placed in the tank at the surface of the water and was provided with a positioning mechanism so that it could be moved relative to the transmitting antenna. The receiving antenna, a small horn, was then located on the top surface of the aluminum sheet and directed toward the transmitting antenna. The receiving horn was movable on the aluminum sheet, therefore the length of the path, $r_{0}+R_{0}$, between the transmitting and receiving antennas could be varied over a substantial range and the proportion of the path which was over water could be varied from about 0.1 to 0.9 . Measurements were made for a number of sets of values of $r_{0}$ and $R_{0}$. The measurements were proportional to the magnitude of the vertical component, $E_{z}$, of the electric field strength at the surface of the aluminum sheet which is related to $F^{\prime}\left(r_{0}+R_{0}, \eta, \eta_{1}\right)$ to a good order of accuracy by

$E_{z}\left(r_{0}+R_{0}, \eta, \eta_{1}\right) \simeq C \frac{e^{-i \beta\left(r_{0}+R_{0}\right)}}{r_{0}+R_{0}} F^{\prime}\left(r_{0}+R_{0}, \eta, \eta_{1}\right)$

where

and

$$
C=-\frac{i \beta I_{b} h_{b}}{2 \pi} \eta_{0}
$$

$$
\eta_{0}=\sqrt{\frac{\mu_{0}}{\epsilon_{0}}}
$$

The experimental measurements were thus proportional to the magnitude of the left-hand side of (12). Let the constant of proportionality be $c$; then the experimental measurements, $\left|A\left(r_{0}+R_{0}, \eta, \eta_{1}\right)\right|$ are given by

$$
\left|A\left(r_{0}+R_{0}, \eta, \eta_{1}\right)\right|=\frac{c|C|}{r_{0}+R_{0}}\left|F^{\prime}\left(r_{0}+R_{0}, \eta, \eta_{1}\right)\right| .
$$

No attempt was made to calculate the value of $c|C|$, but it was determined experimentally. The transmitter and receiver were placed along a path completely over water so that $\eta=\eta_{1}$ and $\left|F^{\prime}\left(r_{0}+R_{0}, \eta, \eta_{1}\right)\right|$ is just the magnitude of the ordinary groundwave attenuation function $\left|F\left(r_{0}+R_{0}, \eta\right)\right|$ as can be obtained from (4). Then a single measurement for a known value of $r_{0}+R_{0}$ gives a value $\left|A\left(r_{0}+R_{0}, \eta, \eta\right)\right|$; and $c|C|$ can be found from this measured value and from $\left|F\left(r_{0}+R_{0}, \eta\right)\right|$ by (13). In practice it was necessary to evaluate $c|\dot{C}|$ frequently because changes in the power line voltage and in the equipment temperature would change the value of $|C|$. Usually several sets of measurements were made for different values of 
$r_{0}+R_{0}$ and the resulting values of $c|C|$ were averaged. After $c|C|$ was determined, (13) was used to evaluate $\left|F^{\prime \prime}\left(r_{0}+R_{0}, \eta, \eta_{1}\right)\right|$ in terms of $r_{0}+R_{0}$ and the measured quantities $\left|A\left(r_{0}+R_{0}, \eta, \eta_{1}\right)\right|$ for cases in which the path is partly over water and partly over the aluminum sheet. The results of the experimental measurements are shown in figures 2 to 6 .

\section{Conclusions}

The experimental data shown in figures 2 to 6 agree reasonably well with the theoretical data calculated from Wait's solution. It is of interest to note the change in the rate at which $\left|F^{\prime}\right|$ decreases with increasing distance. The rate of decrease becomes less when traversing a boundary across which the conductivity increases. In some cases (such as the one considered here) $\left|F^{\prime}\right|$ actually increases with increasing distance for a range of values of distance. This is called the recovery effect; it is clearly evident in both the experimental and the theoretical data. In this investigation the surface to the right of the boundary in figure 1 is assumed to have an infinite conductivity; therefore the recovery effect would be expected to be rather large, and the experimental measurements show this to be true. It would also be expected that $\left|F^{\prime}\right|$ would approach a constant value as distance increases without limit because power losses in the plane can occur only between antenna B and the boundary and this distance $\left(r_{0}\right)$ remains constant as $R_{0}$ increases. The experimental measurements of $\left|F^{\prime}\right|$ do seem to approach a limit as distance increases although this cannot definitely be concluded because of the limited range of distances over which measurements were made.

It may be said that Wait's theory agrees reasonably well with the experimental data in this particular case, and therefore it may be expected that the theory could be applied with good results to other cases of propagation over mixed paths.

\section{References}

Bremmer, H. (1954), The extension of Sommerfeld's formula for the propagation of radio waves over a flat earth to different conductivities of the soil, Physica 20, 441.

Clemmow, P. C. (1953), Radio propagation over a flat earth across a boundary separating two different media, Trans. Roy. Soc. (London) $\mathbf{2 4 6 ,} 1$.

Kirke, H. L. (1949), Calculations of ground wave field strength over a composite land path, Proc. IRE 37, 489.

Millington, G. (1949), Ground wave propagation over an inhomogeneous smooth earth, Part I, Proc. IEE 96, 53.

Norton, K. A. (1936), Propagation of radio waves over the surface of the earth and the upper atmosphere, Proc. IRE 24, No. 10, 1367.

Suda, K. (1954), Field strength calculations-new methods for mixed paths, Wireless Engineer 31, 249.

Wait, J. R. (1956), Mixed path ground wave propagation; 1 short distances, J. Res. NBS 5\%, No. 1, 1-15, RP2687.

Wait, J. R. (1964), Electromagnetic surface waves. Advances in Radio Research, ed. J. A. Saxton, 157-217 (Academic Press, London).

(Paper 68D8-390) 